\title{
Sobrecarga de familiares de pacientes psiquiátricos: comparação entre diferentes tipos de cuidadores
}

\author{
Burden in family caregivers of psychiatric patients: \\ comparison between different types of caregivers \\ Ellen Pinheiro Tenório de Albuquerque', Ana Maria de Oliveira Cintra', Marina Bandeira'
}

\section{RESUMO}

Objetivo: Investigar a sobrecarga objetiva e subjetiva em três diferentes tipos de cuidadores. Método: A Escala de Avaliação da Sobrecarga de Familiares (FBIS-BR) foi aplicada a 30 pais, 30 irmãos e 30 cônjuges de pacientes diagnosticados com transtornos do espectro de esquizofrenia ou transtornos do humor. Resultados: Sete itens apresentaram diferenças significativas entre os grupos. Os pais apresentaram maior sobrecarga do que os irmãos quanto à frequência com que preparavam as refeições dos pacientes e os aconselhavam a ocupar seu tempo livre. Sentiam também maior grau de incômodo por ter que fazer compras para eles e tinham impressão de maior peso financeiro. Comparativamente aos cônjuges, os pais tinham que lidar mais frequentemente com os comportamentos agressivos dos pacientes, tinham maiores gastos com eles e ainda eram mais preocupados com o seu futuro. Já a sobrecarga dos cônjuges foi maior do que a dos irmãos quanto à impressão de peso financeiro. Conclusões: As diferenças encontradas apontam para a necessidade de planejamento de intervenções que considerem as particularidades de cada tipo de cuidador.

\section{ABSTRACT}

Objective: To investigate the subjective and objective burden in three different types of family caregivers. Method: The Family Burden Interview Schedule (FBIS-BR) was administered to $30 \mathrm{pa-}$ rents, 30 siblings and 30 spouses caregivers of psychiatric patients with diagnoses of schizophrenia spectrum or mood disorders. Results: Significant differences between groups were found on seven items of the scale. Parents had higher burden scores than brothers, in regard to how often they prepared meals for patients and advised them on how to use their free time. They also felt a greater degree of discomfort from having to shop for them and had the impression of a greater financial burden. Compared to spouses, parents had to deal more often with patients' aggressive behaviors, had higher expenses with them, and were, in addition, more worried about their future. Spouses had higher burden score than brothers, in regard to perceived financial weight. Conclusions: The differences observed in caregiver burden suggest the need for planning interventions that take into account the particularities of each type of caregiver.

Deinstitutionalization, family burden, kinship.

1 Universidade Federal de São João del-Rei (UFSJ). 


\section{INTRODUÇÃO}

Durante décadas, o tratamento psiquiátrico foi caracterizado por longos períodos de internação e afastamento do doente mental de sua família'. No Brasil, somente em 1980, com a reforma psiquiátrica, esse quadro começou a mudar e a família voltou a fazer parte do cenário de assistência ao paciente ${ }^{1-3}$. Essa mudança marcou uma alteração histórica, na forma como a família vinha participando do cuidado ao doente mental, deixando de ser culpabilizada pela doença e passando a ser considerada uma importante aliada no processo de reabilitação do paciente ${ }^{4,5}$.

No entanto, as pesquisas vêm apontando que as dificuldades enfrentadas pelos familiares no desempenho do papel de cuidador têm contribuído para um sentimento de sobrecarga desses familiares ${ }^{6,7}$. No Brasil, ainda são escassas as pesquisas de avaliação sistemática da sobrecarga, por meio de escalas de medida validadas. Na literatura internacional, são encontrados diversos estudos sobre os fatores que contribuem para maior ou menor sobrecarga familiar e o impacto dessa sobrecarga na saúde mental dos familiares ${ }^{8-10}$. Um fator apontado nas pesquisas, relacionado ao grau de sobrecarga, se refere ao tipo de parentesco do familiar cuidador em relação ao paciente. Embora o número de estudos sobre essa variável seja reduzido, há indicação de que o grau de sobrecarga pode variar em função do tipo de parentesco, sendo necessárias, portanto, pesquisas que investiguem essa variáve $\left.\right|^{6,10-14}$.

Em uma busca nas bases de dados do Medline, Lilacs e SciELO, foram encontrados seis estudos que apontaram a existência de diferenças na sobrecarga sentida pelos familiares cuidadores, em função do tipo de parentesco. Dessas, somente duas tinham como objetivo específico avaliar a influência dessa variável no grau de sobrecarga, comparando grupos de cuidadores ${ }^{11,14}$, enquanto as demais investigaram, em geral, os fatores associados à sobrecarga familiar e, dentre eles, o tipo de parentesco ${ }^{15-19}$.

A escassez de pesquisas relacionadas a essa temática tem impedido o avanço do conhecimento sobre a totalidade dos efeitos da doença mental na família ${ }^{10}$. Visando contribuir para suprir essa lacuna, o presente estudo foi realizado com o objetivo de comparar a sobrecarga sentida por pais, irmãos e cônjuges cuidadores de pacientes psiquiátricos e identificar as dimensões da vida desses familiares mais afetadas pelo papel de cuidador.

\section{MÉTODO}

\section{Delineamento}

Trata-se de um estudo de corte transversal, correlacional, em que foi utilizado um delineamento de comparação com grupo estático ${ }^{20}$ para comparar grupos de pais, irmãos e cônju- ges cuidadores de pacientes psiquiátricos. Para resguardar a validade interna da pesquisa, foi verificada a equivalência dos grupos de familiares quanto às variáveis sociodemográficas e condições de vida dos familiares e às variáveis sociodemográficas e clínicas dos pacientes, visando evitar a interferência dessas variáveis na interpretação dos resultados.

\section{Participantes}

Fizeram parte da pesquisa familiares cuidadores de pacientes psiquiátricos adultos, atendidos em duas instituições psiquiátricas públicas de duas cidades de porte médio de Minas Gerais. Trata-se de uma amostra não probabilística, composta por 90 familiares que atendiam aos critérios de inclusão/ exclusão, definidos para a população-alvo, sendo 30 participantes para cada grupo.

Para os familiares, os critérios de inclusão consistiam em ter mais de 18 anos, conviver diariamente com o paciente e ser indicado como o principal cuidador pelos familiares. Para os pacientes, os critérios de elegibilidade foram: apresentar diagnóstico do espectro de esquizofrenia ou transtornos do humor, segundo critérios da CID-10, conforme indicado nos prontuários, e estar em tratamento regular em uma das instituições psiquiátricas participantes da pesquisa. Foram eliminados os familiares cuidadores de pacientes que estavam internados no período de realização das entrevistas e aqueles que não demonstraram entendimento das perguntas feitas.

\section{Instrumentos de medida}

Para avaliação da sobrecarga, foi utilizada a versão brasileira da escala Family Burden Interview Schedule (FBIS), desenvolvida, originalmente, por Tessler e Gamache ${ }^{21}$ e, posteriormente, adaptada e validada para o Brasil por Bandeira et al. ${ }^{22} \mathrm{e}$ Bandeira et al. ${ }^{23}$, tendo recebido a sigla de FBIS-BR.

A escala FBIS-BR visa avaliar o grau de sobrecarga objetiva e subjetiva apresentado pelos familiares de pacientes psiquiátricos, em cinco dimensões de suas vidas, aferidas em cinco subescalas: A) assistência ao paciente na vida cotidiana, B) supervisão dos comportamentos problemáticos do paciente, C) gastos financeiros relacionados ao paciente, D) impacto na rotina diária da família e E) preocupações com o paciente.

A sobrecarga objetiva é avaliada em termos da frequência de assistências ao paciente na vida cotidiana, de supervisões aos seus comportamentos problemáticos e do impacto na rotina diária do familiar cuidador. A sobrecarga subjetiva é avaliada em termos do grau de incômodo sentido pelo familiar ao realizar as atividades de assistência ao paciente e de supervisão aos comportamentos problemáticos, as frequências de suas preocupações com o paciente, a percepção do familiar com relação às despesas com o paciente e o grau de alterações permanentes em sua vida. A escala FBIS-BR tem qualidades psicométricas adequadas de validade e fidedignidade ${ }^{23}$ e encontra-se disponibilizada no endereço http:// www.ufsj.edu.br/lapsam, na internet.

Um questionário, desenvolvido especificamente para o estudo da sobrecarga de familiares de pacientes psiquiátri- 
cos, foi utilizado para colher os dados sociodemográficos e clínicos dos pacientes. O instrumento foi avaliado por dois especialistas da área de psiquiatria, que indicaram sua adequação ao tema estudado.

\section{Coleta dos dados}

As entrevistas eram individuais, feitas na casa do respondente ou na instituição psiquiátrica. Os encontros eram agendados previamente por telefone, de acordo com a disponibilidade dos familiares. Para cada respondente, as questões eram lidas e as respostas, anotadas por um entrevistador previamente treinado.

\section{Análise de dados}

Para a análise dos dados, foi utilizado o pacote estatístico SPSS, versão 13.0. Inicialmente, os escores de sobrecarga foram submetidos ao teste Kolmogorov-Smirnov, para a verificação de sua distribuição normal. Uma vez confirmada a distribuição normal dos escores de sobrecarga global subjetiva e objetiva, em cada um dos grupos, prosseguiu-se à realização da análise estatística, utilizando testes paramétricos.

Para a caracterização da amostra estudada, foi feita uma análise estatística descritiva, com cálculo das porcentagens, médias, desvios-padrão e valores mínimos e máximos das variáveis sociodemográficas e clínicas. Para evitar variáveis interferentes na comparação dos grupos, visando garantir a validade interna da pesquisa, foi verificada a equivalência dos grupos de cuidadores quanto às características sociodemográficas e condições de vida dos familiares e as características sociodemográficas e clínicas do pacientes, por meio da análise de variância (ANOVA) para variáveis contínuas e o teste qui-quadrado para variáveis categóricas.

Uma análise intragrupo foi feita para avaliar o grau de sobrecarga de cada grupo de cuidadores, separadamente. Utilizou-se o teste $t$ de Student para amostras independentes, a fim de comparar a média dos escores de sobrecarga das subescalas subjetivas A e B. Foram utilizados a análise de variância (ANOVA) e o teste post hoc de Tukey para comparar os escores de sobrecarga objetiva das subescalas A, B e D.

A análise estatística intergrupos, para avaliação das diferenças existentes na sobrecarga dos três grupos de cuidadores, foi feita em três níveis: para os escores globais de sobrecarga objetiva e subjetiva, para cada dimensão da sobrecarga representada nas subescalas e para cada item da escala. Para isso, foram utilizados a análise de variância (ANOVA) e o teste post hoc de Tukey.

\section{Considerações éticas}

A pesquisa foi realizada mediante aprovação pela Comissão de Ética da Universidade Federal de São João del-Rei, conforme memorando número 013/2009/CEPES/UFSJ, e pela coordenação dos serviços participantes. No início das entrevistas, o familiar era informado sobre os objetivos e procedimentos da pesquisa e de que sua participação era voluntária, os da- dos coletados eram de caráter confidencial e a forma de divulgação dos resultados garantiria o sigilo e o anonimato das respostas. Ao final das explicações e do esclarecimento de todas as dúvidas do familiar, era solicitado que o participante assinasse um Termo de Consentimento Livre e Informado.

\section{RESULTADOS}

\section{Descrição da amostra}

As características sociodemográficas da amostra de cuidadores e do quadro clínico dos pacientes se encontram nas tabelas 1 e 2. No grupo de pais, a maioria dos cuidadores $(86,7 \%)$ era do sexo feminino, com idade média de 59,93 anos. Com relação ao pacientes de quem cuidavam, a média de idade era de 31,97 anos e 50\% deles eram do sexo feminino. A maioria $(86,7 \%)$ dos pacientes era solteira e $83,3 \%$ tinham diagnóstico do espectro da esquizofrenia, transtornos esquizotípicos ou delirantes (F 20-29).

No grupo dos irmãos, a maioria dos cuidadores também era do sexo feminino (80\%), com idade média de 45,03 anos. Os pacientes de quem cuidavam tinham a média de idade de 43,89 anos, a maioria era solteira (60\%), do sexo feminino (80\%), com diagnóstico de esquizofrenia, transtornos esquizotípicos ou delirantes (90\%).

Quanto ao grupo de cônjuges, 43,3\% eram do sexo feminino, com idade média de 47,97 anos. Com relação aos pacientes de quem cuidavam, a média de idade era de 45,50 anos, a maioria era do sexo feminino (56,7\%) e também apresentava o diagnóstico de esquizofrenia, transtornos esquizotípicos ou delirantes (73,3\%).

\section{Análise intragrupo da sobrecarga familiar}

Esta análise visou identificar quais eram as dimensões de maior sobrecarga, para cada grupo de cuidadores, separadamente. Os dados comparativos dos escores das subescalas da FBIS-BR se encontram na tabela 3.

No que se refere à sobrecarga objetiva, os cuidadores pais e cônjuges apresentaram maior sobrecarga referente à frequência das assistências prestadas aos pacientes (subescala A objetiva) do que à frequência de supervisões dos seus comportamentos problemáticos (subescala B objetiva). Já no grupo de irmãos, a diferença entre as médias dos escores dessas subescalas não foi estatisticamente significativa.

Comparando-se as subescalas objetivas A e D, pode-se observar que, em todos os grupos, a sobrecarga resultante da frequência de assistência ao paciente (subescala A) foi maior do que a de impacto do papel de cuidador na vida diária do familiar (subescala D). Comparando-se as subescalas B e D, observa-se que, somente no grupo dos cônjuges, a sobrecarga resultante do impacto do papel de cuidador na sua vida diária (subescala D) foi significativamente menor do que a sobrecarga resultante da frequência com a qual o fa- 
Tabela 1. Características sociodemográficas e condições de vida dos familiares, em termos de frequência absoluta e relativa (\%) ou média e desvio-padrão, para cada grupo de cuidador e valores de p referentes à avaliação da equivalência dos grupos, obtidos pelo teste quiquadrado para variáveis categóricas e análise de variância ANOVA, para variáveis contínuas

\begin{tabular}{|c|c|c|c|c|c|}
\hline \multirow[t]{2}{*}{ Variável } & & \multicolumn{3}{|c|}{ Frequências absoluta e relativa (\%)/Média e desvio-padrão (DP) } & \multirow[t]{2}{*}{ p } \\
\hline & & Pais & Irmãos & Cônjuges & \\
\hline Idade & & $59,93(10,04)$ & $45,03(10,17)$ & $47,97(12,93)$ & $0,00^{* 0}$ \\
\hline \multirow[t]{2}{*}{ Sexo } & Masculino & $4(13,3 \%)$ & $6(20,0 \%)$ & $17(56,7 \%)$ & $0,00^{* \mathbf{\Delta}}$ \\
\hline & Feminino & $26(86,7 \%)$ & $24(80,0 \%)$ & $13(43,3 \%)$ & \\
\hline \multirow[t]{4}{*}{ Estado civil } & Solteiro & $1(3,3 \%)$ & $18(60,0 \%)$ & - & $0,00^{* \Delta}$ \\
\hline & Casado & $13(43,3 \%)$ & $11(36,7 \%)$ & $(100 \%)$ & \\
\hline & Separado ou divorciado & $5(16,7 \%)$ & - & - & \\
\hline & Viúvo & $11(36,7 \%)$ & $1(3,3 \%)$ & - & \\
\hline \multirow[t]{6}{*}{ Escolaridade } & Analfabeto & $5(16,7 \%)$ & - & $3(10,0 \%)$ & $0,25 \mathbf{\Delta}$ \\
\hline & $1^{\circ}$ grau incompleto & $24(80,0 \%)$ & $22(73,3 \%)$ & $18(60,0 \%)$ & \\
\hline & $1^{\circ}$ grau completo & $1(3,3 \%)$ & $4(13,3 \%)$ & $4(13,3 \%)$ & \\
\hline & $2^{\circ}$ grau incompleto & - & $2(6,7 \%)$ & $2(6,7 \%)$ & \\
\hline & $2^{\circ}$ grau completo & - & $1(3,3 \%)$ & $2(6,7 \%)$ & \\
\hline & $3^{\circ}$ grau completo & - & $1(3,3 \%)$ & $1(3,3 \%)$ & \\
\hline \multirow[t]{2}{*}{ Está trabalhando } & Sim & $7(23,3 \%)$ & $17(56,7 \%)$ & $16(53,3 \%)$ & $0,02^{* \Delta}$ \\
\hline & Não & $23(76,7 \%)$ & $13(43,3 \%)$ & $14(46,7 \%)$ & \\
\hline \multirow[t]{6}{*}{ Valor da renda mensal } & Nenhum & $6(20,0 \%)$ & $9(31,0 \%)$ & $10(34,5 \%)$ & $0,5^{\mathbf{\Lambda}}$ \\
\hline & Menos de 1 salário-mínimo & $4(13,3 \%)$ & $4(13,8 \%)$ & $2(6,9 \%)$ & \\
\hline & De 1 a 2 salários-mínimos & $15(50,0 \%)$ & $14(48,3 \%)$ & $14(48,3 \%)$ & \\
\hline & De 2,1 a 3 salários-mínimos & $3(10,0 \%)$ & $1(3,4 \%)$ & $1(3,4 \%)$ & \\
\hline & De 3,1 a 4 salários-mínimos & - & $1(3,4 \%)$ & $2(6,9 \%)$ & \\
\hline & Mais de 4 salários-mínimos & $2(6,7 \%)$ & - & - & \\
\hline \multirow[t]{2}{*}{ Cuida de outro familiar doente } & Sim & $16(53,3 \%)$ & $19(63,3 \%)$ & $13(43,3 \%)$ & $0,30^{\mathbf{4}}$ \\
\hline & Não & $14(46,7 \%)$ & $11(36,7 \%)$ & $17(56,7 \%)$ & \\
\hline \multirow[t]{3}{*}{ Tem problema de saúde } & Sim & $15(50,0 \%)$ & $14(58,3 \%)$ & $12(85,7 \%)$ & $0,18^{\mathbf{4}}$ \\
\hline & Não & $5(16,7 \%)$ & $10(41,7 \%)$ & $2(14,3 \%)$ & \\
\hline & Não responderam & $10(33,3 \%)$ & & & \\
\hline \multirow[t]{2}{*}{ Faz tratamento de saúde } & Sim & $14(70,0 \%)$ & $12(50,0 \%)$ & $8(57,1 \%)$ & $0,40^{\mathbf{4}}$ \\
\hline & Não & $6(30,0 \%)$ & $12(50,0 \%)$ & $6(42,9 \%)$ & \\
\hline \multirow{3}{*}{$\begin{array}{l}\text { Recebe ajuda para cuidar do } \\
\text { paciente }\end{array}$} & Sempre & $16(53,3 \%)$ & $20(66,7 \%)$ & $14(46,7 \%)$ & $0,37^{\mathbf{4}}$ \\
\hline & Às vezes & $6(20,0 \%)$ & $2(6,7 \%)$ & $4(13,3 \%)$ & \\
\hline & Nunca & $8(26,7 \%)$ & $8(26,7 \%)$ & $12(40,0 \%)$ & \\
\hline \multirow[t]{4}{*}{ Tipo de ajuda } & Nas tarefas & $8(26,7 \%)$ & $6(20,0 \%)$ & $12(40,0 \%)$ & $0,13^{\star}$ \\
\hline & Financeira e em tarefas & $9(30,0 \%)$ & $12(40,0 \%)$ & $6(20,0 \%)$ & \\
\hline & Financeira & $5(16,7 \%)$ & $4(13,3 \%)$ & - & \\
\hline & Nenhum tipo de ajuda & $8(26,7 \%)$ & $8(26,7 \%)$ & $12(40,0 \%)$ & \\
\hline \multirow[t]{2}{*}{ Está satisfeito com tratamento } & $\operatorname{Sim}$ & $26(86,7 \%)$ & $26(86,7 \%)$ & $24(82,8 \%)$ & $0,89^{\mathbf{4}}$ \\
\hline & Não & $4(13,3 \%)$ & $4(13,3 \%)$ & $5(17,2 \%)$ & \\
\hline
\end{tabular}

*:p $<0,05 ; \mathbf{\Lambda}$ : teste qui-quadrado; $\mathbf{\mathbf { A }}$ ANOVA; DP: desvio-padrão.

miliar supervisionou os comportamentos problemáticos do paciente (subescala B).

Os resultados da sobrecarga subjetiva mostraram que, para cada grupo, os familiares sentiram maior grau de incô- modo ao supervisionar os comportamentos problemáticos dos pacientes (subescala B) do que ao fazer tarefas cotidianas de assistência (subescala A), embora os comportamentos problemáticos ocorressem em menor frequência. 
Tabela 2. Características sociodemográficas e clínicas dos pacientes, em termos de frequência absoluta e relativa (\%) ou média e desviopadrão (DP) para cada grupo de cuidador e valores de p referentes à avaliação da equivalência dos grupos, obtidos pelo teste quiquadrado para variáveis categóricas e análise de variância ANOVA, para variáveis contínuas

\begin{tabular}{|c|c|c|c|c|c|}
\hline \multicolumn{2}{|c|}{ Características sociodemográficas e clínicas do paciente } & \multicolumn{3}{|c|}{ Frequências absoluta e relativa (\%)/Média e desvio-padrão (DP) } & \multirow[t]{2}{*}{$\mathrm{p}$} \\
\hline & & \multirow{2}{*}{$\begin{array}{l}\text { Pais } \\
31,97(9,20)\end{array}$} & \multirow{2}{*}{$\begin{array}{l}\text { Irmãos } \\
43,89(10,29)\end{array}$} & \multirow{2}{*}{$\begin{array}{l}\text { Cônjuges } \\
45,50(11,81)\end{array}$} & \\
\hline Idade & & & & & $0,00^{*}$ \\
\hline \multirow[t]{2}{*}{ Sexo } & Masculino & $15(50,0 \%)$ & $14(46,7 \%)$ & $13(43,3 \%)$ & $0,87^{\Perp}$ \\
\hline & Feminino & $15(50,0 \%)$ & $16(53,3 \%)$ & $17(56,7 \%)$ & \\
\hline \multirow[t]{3}{*}{ Estado civil } & Solteiro & $26(86,7 \%)$ & $26(86,7 \%)$ & & $0,00^{*}$ \\
\hline & Casado & $1(3,3 \%)$ & $2(6,7 \%)$ & $30(100 \%)$ & \\
\hline & Separado ou divorciado & $3(10,0 \%)$ & $2(6,7 \%)$ & - & \\
\hline \multirow[t]{6}{*}{ Escolaridade } & Analfabeto & $3(10,0 \%)$ & $2(6,9 \%)$ & $4(13,3 \%)$ & $0,30^{\mathbf{4}}$ \\
\hline & $1^{\circ}$ grau incompleto & $15(50,0 \%)$ & $23(79,3 \%)$ & $20(66,7 \%)$ & \\
\hline & $1^{\circ}$ grau completo & $6(20,0 \%)$ & $2(6,9 \%)$ & $1(3,3 \%)$ & \\
\hline & $2^{\circ}$ grau incompleto & $1(3,3 \%)$ & - & $2(6,7 \%)$ & \\
\hline & $2^{\circ}$ grau completo & $4(13,3 \%)$ & $1(3,4 \%)$ & $3(10,0 \%)$ & \\
\hline & $3^{\circ}$ grau incompleto & $1(3,3 \%)$ & $1(3,4 \%)$ & - & \\
\hline \multicolumn{2}{|l|}{ Instituição } & & & & $0,00^{*}$ \\
\hline \multicolumn{2}{|l|}{ CAPS - Lavras } & $15(50,0 \%)$ & $23(76,7 \%)$ & $6(20,0 \%)$ & \\
\hline \multicolumn{2}{|l|}{ CAPS - São João del-Rei } & $15(50,0 \%)$ & $7(23,3 \%)$ & $24(80,0 \%)$ & \\
\hline \multicolumn{2}{|l|}{ Grupo Diagnóstico CID } & & & & 0,24 \\
\hline \multicolumn{2}{|c|}{ Transtornos do espectro esquizofrênico (F 20-29) } & $25(83,3 \%)$ & $27(90,0 \%)$ & $22(73,3 \%)$ & \\
\hline \multicolumn{2}{|c|}{ Transtornos do humor (F 30-39) } & $5(16,7 \%)$ & $3(10,0 \%)$ & $8(26,7 \%)$ & \\
\hline \multirow[t]{2}{*}{ Outros transtornos } & Sim & $8(26,7 \%)$ & $5(16,7 \%)$ & $4(13,3 \%)$ & $0,39 \mathbf{4}$ \\
\hline & Não & $22(73,3 \%)$ & $25(83,3 \%)$ & $26(86,7 \%)$ & \\
\hline \multirow[t]{2}{*}{ Doenças físicas } & Sim & $10(33,3 \%)$ & $14(46,7 \%)$ & $19(63,3 \%)$ & $0,07^{\Perp}$ \\
\hline & Não & $20(66,7 \%)$ & $16(53,3 \%)$ & $11(36,7 \%)$ & \\
\hline \multirow[t]{2}{*}{ Presença de crise atual } & Sim & $6(20,0 \%)$ & $5(16,7 \%)$ & $10(33,3 \%)$ & $0,27^{\wedge}$ \\
\hline & Não & $24(80,0 \%)$ & $25(83,3 \%)$ & $20(66,7 \%)$ & \\
\hline \multicolumn{2}{|c|}{ Número de crises no último ano } & $14,10(33,65)$ & $6,41(11,75)$ & $4,65(12,32)$ & $0,29^{a}$ \\
\hline \multicolumn{2}{|l|}{ Número total de internações } & $5,53(18,69)$ & $10,76(13,48)$ & $5,56(23,95)$ & $0,49^{\circ}$ \\
\hline \multicolumn{2}{|c|}{ Tempo total de internação, em anos } & $1,01(3,05)$ & $4,43(7,65)$ & $1,22(4,33)$ & $0,03^{* 0}$ \\
\hline \multicolumn{2}{|l|}{ Duração, em anos, da doença } & $15,25(10,08)$ & $22,28(14,24)$ & $12,71(10,29)$ & $0,008^{* a}$ \\
\hline \multicolumn{2}{|c|}{ Duração, em anos, do tratamento psiquiátrico } & $12,64(9,06)$ & $16,73(12,07)$ & $7,95(8,15)$ & $0,004^{* a}$ \\
\hline \multicolumn{2}{|l|}{ Número de remédios } & $3,93(1,26)$ & $3,52(1,43)$ & $3,33(1,09)$ & $0,18^{\circ}$ \\
\hline \multirow[t]{3}{*}{ Aceitação da medicação } & Sim & $23(76,7 \%)$ & $22(73,3 \%)$ & $27(90 \%)$ & 0,28 \\
\hline & Não & $1(3,3 \%)$ & $3(10,0 \%)$ & $0(0 \%)$ & \\
\hline & Às vezes & $6(20,0 \%)$ & $5(16,7 \%)$ & $3(10,0 \%)$ & \\
\hline \multirow[t]{2}{*}{ Toma a medicação sozinho } & Sim & $13(43,3 \%)$ & $14(46,7 \%)$ & $13(43,3 \%)$ & $0,96^{\mathbf{4}}$ \\
\hline & Não & $17(56,7 \%)$ & $16(53,3 \%)$ & $17(56,7 \%)$ & \\
\hline
\end{tabular}

*: $p<0,05 ; \mathbf{\Lambda}$ : teste qui-quadrado; $\mathbf{\square}:$ ANOVA; DP: desvio-padrão.

Tabela 3. Análise intragrupo das diferenças entre as médias das subescalas subjetivas A e B da FBIS-BR, pelo teste t de Student, e entre as médias das subescalas objetivas A, B e D, pela análise de variância (ANOVA) com teste post hoc de Tukey, desvios-padrão (DP) e valor de p, para os grupos de Pais, Irmãos e Cônjuges

\begin{tabular}{|c|c|c|c|c|c|c|c|c|c|c|}
\hline \multirow[t]{3}{*}{ Familiar } & \multirow{2}{*}{\multicolumn{3}{|c|}{$\begin{array}{c}\text { Médias (DP) } \\
\text { Subescalas objetivas }\end{array}$}} & \multirow{3}{*}{$\begin{array}{c}\text { ANOVA } \\
p\end{array}$} & \multicolumn{3}{|c|}{ Post hoc de Tukey } & \multirow{2}{*}{\multicolumn{2}{|c|}{$\begin{array}{c}\text { Médias (DP) } \\
\text { Subescalas subjetivas }\end{array}$}} & \multirow[t]{3}{*}{ Tde Student } \\
\hline & & & & & \multirow[t]{2}{*}{$p(A B)$} & \multirow[t]{2}{*}{$p(A D)$} & \multirow[t]{2}{*}{$p(B D)$} & & & \\
\hline & $A$ & B & D & & & & & $A$ & B & \\
\hline Pais & $3,14(0,72)$ & $2,35(0,96)$ & $1,79(1,06)$ & $0,00^{* *}$ & $0,004^{*}$ & $0,00^{* *}$ & 0,06 & $1,85(0,77)$ & $3,05(0,99)$ & $0,00^{* *}$ \\
\hline Irmãos & $2,61(1,00)$ & $2,12(0,83)$ & $1,61(0,86)$ & $0,00^{* *}$ & 1,00 & $0,00^{* *}$ & 0,08 & $1,66(0,79)$ & $3,03(0,90)$ & $0,00^{* *}$ \\
\hline Cônjuges & $2,79(0,83)$ & $2,10(0,78)$ & $1,47(0,63)$ & $0,00^{* *}$ & $0,002^{*}$ & $0,00 * *$ & $0,004^{*}$ & $1,75(0,67)$ & $3,28(0,78)$ & $0,00^{* *}$ \\
\hline
\end{tabular}

*: $p<0,05 ; * *: p \leq 0,001 ; D P$ : desvio-padrão; AB: subescalas A e B; AD: subescalas A e B; BD: subescalas B e D. 


\section{Análise intergrupo da sobrecarga familiar}

Equivalência dos grupos: os resultados mostraram que os três grupos de cuidadores eram equivalentes $(p>0,05) \mathrm{com}$ relação às seguintes variáveis dos familiares: escolaridade, valor da renda, cuidar de outro familiar doente, ter problema de saúde, fazer tratamento de saúde, receber ajuda para cuidar do paciente, tipo de ajuda recebida e satisfação com o tratamento recebido. Entretanto, os grupos não foram equivalentes com relação às seguintes variáveis dos familiares que podem interferir com o grau de sobrecarga: idade, sexo, estado civil e situação de trabalho (Tabela 1).

Com relação às características sociodemográficas e clínicas dos pacientes, os grupos eram equivalentes $(p>0,05)$ quanto às seguintes variáveis: sexo, escolaridade, diagnósti$\mathrm{co}$, presença de comorbidade, presença de doenças físicas, estar em crise no período em que foi realizada a entrevista, número de crises no ano anterior à entrevista, número total de internações, número de remédios, aceitação da medicação e capacidade de tomar os remédios sozinho. Entretanto, os grupos não eram equivalentes com relação às seguintes variáveis dos pacientes que podem interferir com o grau de sobrecarga do cuidador: idade, estado civil, local de tratamento, tempo total de internação e duração da doença (Tabela 2).

Análise dos escores globais e das subescalas de sobrecarga: na tabela 4 são apresentados os resultados da comparação entre os três grupos de cuidadores, com relação ao grau de sobrecarga global objetiva e global subjetiva. Não houve diferença estatisticamente significativa entre as médias da sobrecarga global objetiva e subjetiva entre os grupos. Na tabela 4 também são apresentados os resultados da comparação dos três grupos quanto aos escores das subescalas. Os dados indicam que os familiares também não diferiram quanto aos escores médios de sobrecarga nas dimensões avaliadas pelas subescalas.
Análise por itens: na tabela 5, são apresentados os resultados da análise comparativa, por itens, da sobrecarga dos três grupos de cuidadores. Na subescala A, referente à assistência ao paciente na vida cotidiana, a sobrecarga objetiva dos pais foi mais elevada do que a dos irmãos, no que se refere a dois aspectos: a frequência com que o familiar preparou ou ajudou o paciente a preparar as suas refeições (questão A5a) e a frequência com que o familiar teve que lembrar ou insistir com o paciente para que ele ocupasse seu tempo livre com alguma coisa (questão A8a). Ainda na subescala A, a avaliação da sobrecarga subjetiva mostrou que o grau de incômodo sentido pelos cuidadores ao terem que fazer compras de alimentos, roupas ou outros objetos (questão A4b) para o paciente foi maior no grupo de pais do que no grupo de irmãos.

Com relação à subescala $B$, referente à supervisão dos comportamentos problemáticos dos pacientes, um item apresentou diferença estatisticamente significativa entre $\mathrm{o}$ grupo de pais e cônjuges. No grupo dos pais, a sobrecarga objetiva foi mais elevada do que a dos cônjuges, em termos da frequência com que o familiar teve que lidar ou tentou evitar/impedir que o paciente fosse agressivo com outras pessoas (questão B4a).

Na subescala C, que indica o impacto financeiro na vida do cuidador, observa-se que os gastos dos pais com o paciente foram significativamente superiores aos dos cônjuges. Os pais gastaram, em média, R\$181,10, enquanto a média dos gastos dos cônjuges cuidadores foi de $\mathrm{R} \$ 80,95$. Com relação ao sentimento de peso financeiro decorrente dos gastos com o paciente no último ano, o grupo de pais teve uma sobrecarga maior do que o grupo de irmãos e, comparativamente aos cônjuges, os irmãos também apresentaram menor sentimento de peso relacionado aos gastos.

Na subescala $E$, referente às preocupações do cuidador com o paciente, os pais apresentaram sobrecarga subjetiva significativamente mais elevada do que os cônjuges, ao se preocuparem com o futuro do paciente (questão E7).

Tabela 4. Médias dos escores globais e das subescalas de sobrecarga objetiva e subjetiva, medidos pela escala FBIS-BR, desvios-padrão (DP) e valor de p para os grupos de Pais, Irmãos e Cônjuges, avaliados pela análise de variância (ANOVA) e teste post hoc de Tukey

\begin{tabular}{|c|c|c|c|c|c|c|c|c|}
\hline \multirow[t]{2}{*}{ Escala/subescala } & & \multicolumn{3}{|c|}{ Médias (DP) } & \multirow{2}{*}{$\begin{array}{c}\text { ANOVA } \\
\mathbf{p}\end{array}$} & \multicolumn{3}{|c|}{ Posthoc de Tukey } \\
\hline & & Pais & Irmãos & Cônjuges & & $\mathrm{p}(\mathrm{PI})$ & $\mathrm{p}(\mathrm{PC})$ & $\mathrm{p}(\mathrm{IC})$ \\
\hline Sobrecarga global objetiva & & $2,60(0,70)$ & $2,25(0,78)$ & $2,29(0,58)$ & 0,10 & 0,12 & 0,19 & 0,97 \\
\hline Sobrecarga global subjetiva & & $2,91(0,49)$ & $2,73(0,66)$ & $2,82(0,53)$ & 0,46 & 0,42 & 0,79 & 0,82 \\
\hline \multirow[t]{2}{*}{ A - Assistência na vida cotidiana } & Sobrecarga objetiva & $3,14(0,72)$ & $2,61(0,99)$ & $2,78(0,83)$ & 0,04 & 0,05 & 0,25 & 0,71 \\
\hline & Sobrecarga subjetiva & $1,85(0,77)$ & $1,66(0,79)$ & $1,75(0,67)$ & 0,69 & 0,59 & 0,85 & 0,90 \\
\hline \multirow[t]{2}{*}{ B - Supervisão dos comportamentos problemáticos } & Sobrecarga objetiva & $2,35(0,96)$ & $2,12(0,83)$ & $2,10(0,78)$ & 0,41 & 0,56 & 0,50 & 0,99 \\
\hline & Sobrecarga subjetiva & $3,05(0,99)$ & $3,03(0,90)$ & $3,28(0,78)$ & 0,13 & 0,99 & 0,60 & 0,56 \\
\hline D- Impacto na rotina & Sobrecarga objetiva & $1,79(1,06)$ & $1,61(0,86)$ & $1,47(0,63)$ & 0,05 & 0,69 & 0,32 & 0,80 \\
\hline E - Preocupações com o paciente & Sobrecarga subjetiva & $3,80(0,65)$ & $3,48(0,90)$ & $3,58(0,92)$ & 0,31 & 0,30 & 0,56 & 0,89 \\
\hline
\end{tabular}

DP: desvio-padrão; PI: pais e irmãos; PC: pais e cônjuges; IC: irmãos e cônjuges. 
Tabela 5. Médias dos escores de itens da FBIS-BR, desvios-padrão (DP) e valor de p para as diferenças entre os grupos de Pais, Irmãos e Cônjuges, avaliadas pela análise de variância (ANOVA) e teste post hoc de Tukey

\begin{tabular}{|c|c|c|c|c|c|c|c|}
\hline \multirow[t]{2}{*}{ Item } & \multicolumn{3}{|c|}{ Médias (DP) } & \multirow{2}{*}{$\begin{array}{c}\text { ANOVA } \\
\mathbf{p}\end{array}$} & \multicolumn{3}{|c|}{ Posthocde Tukey } \\
\hline & Pais & Irmäos & Cônjuges & & $\mathrm{p}(\mathrm{PI})$ & $\mathrm{p}(\mathrm{PC})$ & $p(I C)$ \\
\hline Incômodo em fazer compras (A4b) & $2,17(1,27)$ & $1,17(0,71)$ & $1,63(0,46)$ & $0,01^{*}$ & $0,008^{*}$ & 0,24 & 0,41 \\
\hline Frequência de preparo de refeições ( $\mathrm{A} 5 \mathrm{a}$ ) & $4,50(1,14)$ & $3,33(1,92)$ & $3,63(1,67)$ & $0,02^{*}$ & $0,02^{*}$ & 0,09 & 0,75 \\
\hline Ocupar tempo (A8a) & $4,00(1,39)$ & $2,90(1,56)$ & $3,37(1,77)$ & $0,03^{*}$ & $0,02^{*}$ & 0,27 & 0,49 \\
\hline Comportamento agressivo (B4a) & $2,30(1,68)$ & $1,60(1,00)$ & $1,27(0,58)$ & $0,004^{*}$ & 0,06 & $0,003^{*}$ & 0,52 \\
\hline Gastos com o paciente (C4) & $181,10(185,92)$ & $169,69(172,99)$ & $80,95(109,01)$ & $0,03^{*}$ & 0,96 & $0,04^{*}$ & 0,09 \\
\hline Impressão do peso dos gastos com o paciente no último ano (C5) & $3,17(1,62)$ & $1,67(1,35)$ & $2,80(1,69)$ & $0,001^{* *}$ & $0,001^{* *}$ & 0,63 & $0,02^{*}$ \\
\hline Preocupação com o futuro do paciente (E7) & $4,90(0,55)$ & $4,33(0,99)$ & $4,27(1,30)$ & $0,03^{*}$ & 0,08 & $0,04^{*}$ & 0,96 \\
\hline
\end{tabular}

*: $p<0,05 ;{ }^{* *}: p \leq 0,001 ;$ DP: desvio-padrão; PI: pais e irmãos; PC: pais e cônjuges; IC: irmãos e cônjuges

\section{DISCUSSÃO}

Os resultados deste estudo possibilitaram identificar semeIhanças e diferenças entre pais, irmãos e cônjuges cuidadores de pacientes psiquiátricos, com relação à sobrecarga resultante do papel de cuidador. Com relação às semelhanças encontradas entre os três grupos, constatou-se que as maiores médias de sobrecarga objetiva se concentravam na subescala $A$, referente à frequência de assistência ao paciente na vida cotidiana. Essa frequência foi superior à da supervisão dos comportamentos problemáticos dos pacientes (subescala B), para cada grupo de cuidador. Apesar disso, os três grupos apresentaram escores mais elevados de sobrecarga subjetiva, com maior sentimento de incômodo, ao supervisionar os comportamentos problemáticos dos pacientes do que ao prestar assistência nas tarefas diárias. Esses resultados foram similares aos dados das pesquisas de Barroso et al. ${ }^{24} \mathrm{e}$ de Albuquerque et al. ${ }^{14}$, nas quais os familiares se sentiram muito incomodados por supervisionar os comportamentos problemáticos dos pacientes, apesar de serem menos frequentes, e se sentiram pouco incomodados ao auxiliar o paciente em suas tarefas cotidianas.

Os grupos não diferiram com relação aos graus de sobrecarga global objetiva e subjetiva, nem quanto à sobrecarga por domínios da vida dos cuidadores. Esses resultados indicam que, de maneira geral, o papel de cuidador afetou os três grupos estudados de forma semelhante. Esses dados corroboram resultados encontrados por Jungbauer e Angermeyer ${ }^{11}$, que também encontraram algumas semelhanças na sobrecarga sentida por cônjuges e pais cuidadores, por exemplo, a variação da sobrecarga de acordo com a fase da doença do paciente.

No entanto, a análise comparativa, por itens, dos três grupos de cuidadores colocou em destaque as diferenças entre eles, quanto a aspectos específicos da sobrecarga. Os resultados mostraram que os pais apresentavam maior grau de sobrecarga do que os irmãos e/ou os cônjuges, em um total de sete itens. Esses resultados corroboram dados en- contrados na literatura, indicando que os pais, além de serem apontados como os principais cuidadores dos pacientes psiquiátricos, também são os que apresentam sobrecarga mais elevada ${ }^{14,15,19,25}$. Segundo Jungbauer e Angermeyer ${ }^{11}$, os cuidadores são confrontados com uma sobrecarga específica, pautada pelo tipo de relacionamento que têm com o paciente. Nesse sentido, o grau de parentesco que o cuidador tem com o paciente determina as especificidades do relacionamento entre eles, resultando em diferenças específicas na sobrecarga.

A comparação entre a sobrecarga de pais e de irmãos demonstrou que os pais apresentaram maior sobrecarga objetiva do que os irmãos quanto à frequência com que o familiar preparou ou ajudou o paciente a preparar as suas refeições e a frequência com que o familiar teve que lembrar ou insistir com o paciente para que ele ocupasse seu tempo livre com alguma coisa. No entanto, a sobrecarga subjetiva, ou o incômodo referente a esses itens, não apresentou diferença significativa entre os grupos.

Porém, com relação ao aspecto financeiro, os pais apresentaram maior sentimento de incômodo do que os irmãos, no item referente a fazer compras de alimentos, roupas ou outros objetos para o paciente. Uma possível explicação para esse incômodo pode estar relacionada a dificuldades financeiras enfrentadas pelas famílias, uma vez que a sobrecarga dos pais também foi maior do que a dos irmãos, no item referente à impressão do peso dos gastos com o paciente no último ano. Essa diferença também pode ser observada na comparação entre irmãos e cônjuges, pois os cônjuges tiveram impressão de peso financeiro maior do que a dos irmãos. Na pesquisa de Albuquerque et al. ${ }^{14}$, o grau de sobrecarga subjetiva dos pais, ou seja, o incômodo sentido por eles, também foi mais acentuado do que o dos irmãos, ao fazerem compras para o paciente e ao relatarem o sentimento de sobrecarga financeira com relação aos gastos feitos com o paciente, no último ano.

Comparando-se pais e cônjuges, algumas diferenças também puderam ser apontadas. A primeira ainda se refere 
às questões financeiras, tendo sido constatado que os pais tinham gastos significativamente superiores aos dos cônjuges. Esse resultado do aspecto financeiro da sobrecarga deve ser colocado em destaque, tendo em vista que, das oito áreas identificadas por Tessler e Gamache ${ }^{7}$ que compõem a dimensão do cuidar, a administração do dinheiro do paciente foi aquela em que os familiares perceberam que precisavam dar maior assistência aos pacientes. No estudo de Barroso ${ }^{19}$, que analisou os fatores associados à sobrecarga, esse aspecto também foi destacado, pois foi um dos fatores que mais contribuíram para a sobrecarga objetiva dos familiares, em uma análise de regressão múltipla. Na pesquisa de Martínez et al. ${ }^{15}$, os gastos com o paciente e as preocupações geradas por dificuldades financeiras também foram maiores para os pais e irmãos, comparativamente aos cônjuges. Diante disso, a sobrecarga financeira parece ser uma questão que não deve ser negligenciada pelos profissionais, ao trabalharem com essa população de cuidadores de pacientes psiquiátricos.

Outras diferenças foram identificadas quanto à sobrecarga de pais e cônjuges cuidadores. A frequência com que os pais precisaram lidar ou tentaram impedir que o paciente insultasse, ameaçasse ou machucasse alguém foi mais elevada do que no grupo de cônjuges. No entanto, não foram observadas diferenças significativas entre esses dois grupos quanto ao sentimento de incômodo resultante desse comportamento problemático do paciente.

A preocupação com o futuro do paciente também foi significativamente mais elevada no grupo de pais do que de cônjuges, embora essa diferença não tenha sido significativa entre os demais grupos. Esses resultados corroboram os achados do estudo de Albuquerque et al.14, que também não observaram diferenças significativas entre pais e irmãos no que se refere, especificamente, a esse item da escala FBIS-BR. No entanto, essa parece ser uma questão que merece ser estudada com mais profundidade, uma vez que dados da literatura apontam os pais como os principais cuidadores e destacam a preocupação deles em relação ao futuro do filho doente. A idade mais avançada, as limitações no tipo de tarefas para cuidar do paciente e o medo de não estarem presentes por muito tempo ao lado do filho resultam em uma grande fonte de preocupação para esses cuidadores $11,15,16,26$.

Os resultados deste estudo permitiram corroborar alguns dados da literatura, contribuindo para consolidar o conhecimento sobre o tema. No entanto, destaca-se a dificuldade de comparar certas dimensões da sobrecarga sentida pelos cuidadores com os resultados apresentados por outras pesquisas nacionais e internacionais, uma vez que elas não focalizaram a relação do parentesco do cuidador.

Outra dificuldade na discussão dos dados pode ser atribuída à ausência de uma padronização dos instrumentos de avaliação utilizados pelas pesquisas, necessária para a comparação dos resultados ${ }^{27,28}$. Além disso, em muitos estudos, os resultados foram registrados de forma descritiva, havendo carência de dados estatísticos, tal como apontado por Bandeira e Barroso ${ }^{10}$.

Uma limitação do presente estudo foi o fato de a amostra não ter sido selecionada aleatoriamente da população-alvo, o que restringe a possibilidade de generalização dos resultados. Além disso, não foi computado o número de sujeitos eliminados em função dos critérios de exclusão, o que pode ter limitado a composição da amostra. Outra limitação foi a impossibilidade de igualar os grupos com relação a todas as variáveis analisadas. Algumas diferenças na composição dos grupos eram esperadas, por serem características inerentes a cada grupo de cuidadores como a idade e a situação de trabalho dos familiares. Essas variáveis podem interagir com a variável do relacionamento com o paciente, modulando, assim, a experiência do papel de cuidador, típica de cada grupo. Além disso, algumas diferenças entre os grupos que não foram estatisticamente significativas podem, no entanto, ter relevância clínica, podendo ter interferido nos resultados da sobrecarga. Estudos futuros poderão elucidar essas questões, utilizando delineamentos de pesquisa que permitam maior equivalência dos grupos.

Por fim, destaca-se a importância de ampliar o estudo da sobrecarga de outros tipos de familiares cuidadores, tais como filhos e parentes por afinidade do paciente (ex:: genros e enteados), que não foram analisados no presente trabalho. Dados sobre esses grupos de cuidadores ainda são escassos, mesmo na literatura internacional. Também não foi analisada a influência do gênero na sobrecarga dos cuidadores. Estudos futuros poderão investigar a influência dessas variáveis na sobrecarga dos cuidadores, permitindo, assim, o aprofundamento do conhecimento sobre essa temática.

\section{CONCLUSÃO}

Os resultados desta pesquisa permitiram constatar e contrastar a sobrecarga de pais, irmãos e cônjuges cuidadores. Observou-se que a doença mental atinge não só o paciente, mas toda a sua família, que parece não estar superando as exigências e as dificuldades resultantes do papel de cuidador. Essa situação, além de poder ter reflexos negativos no cuidado prestado ao próprio paciente, tem o potencial, como afirmou Doornbos ${ }^{16}$, para criar um novo cliente para os serviços de saúde mental: o próprio familiar cuidador.

De forma mais específica, com esta pesquisa oferece-se uma contribuição para um melhor entendimento da sobrecarga de familiares cuidadores de pacientes psiquiátricos, uma vez que foi constatada a existência de algumas diferenças entre os grupos analisados, com relação à sobrecarga sentida. Esses dados são importantes, pois apontam que diferentes tipos de parentesco estão relacionados a níveis distintos de sobrecarga, o que sugere a necessidade de planejamento de intervenções específicas para cada tipo de cuidador, visando à redução da sobrecarga familiar. 


\section{AGRADECIMENTO}

Este artigo faz parte de um projeto de pesquisa financiado pela Fundação de Amparo à Pesquisa do Estado de Minas Gerais (Fapemig) e pelo Conselho Nacional de Desenvolvimento Científico e Tecnológico (CNPq).

Agências financiadoras: Fapemig e CNPq.

Conflito de interesses: nenhum.

\section{REFERÊNCIAS}

1. Waidman MAP, Jouclas VMG, Stefanelli MC. Família e doença mental. Família, Saúde e Desenvolvimento. 1999;1(1/2):27-32.

2. Moreno $V$, Alencastre MB. A trajetória da família do portador de sofrimento psíquico. Rev Esc Enferm USP. 2003;37(2):43-50.

3. Moreno $V$, Alencastre MB. A família do portador de sofrimento psíquico e os serviços de saúde mental: estudo de caso. Acta Sci., Health Sci. 2004;26(1):175-81.

4. Yacubian J, Neto FL. Psicoeducação familiar. Família, Saúde e Desenvolvimento. 2001;3(2):98-108

5. Pereira MAO, Pereira Jr A. Transtorno mental: dificuldades enfrentadas pela família. Rev Esc Enferm USP. 2003;37(4):92-100.

6. Maurin JT, Boyd CB. Burden of mental illness on the family: a critical review. Arch Psychiatr Nurs. 1990;4(2):99-107.

7. Tessler RC, Gamache GM. Family experiences with mental illness. Auburn House: Westport; 2000.

8. Loukissa AD. Family burden in chronic mental illness: a review of research studies. J Adv Nursing. 1995;21(2):248-55

9. Rose LE. Families of psychiatric patients: a critical review and future research directions. Arch Psychiatr Nurs. 1996;10(2):67-76.

10. Bandeira M, Barroso S. A sobrecarga das famílias de pacientes psiquiátricos. J Bras Psiquiatr. 2005;54(1):34-46

11. Jungbauer J, Angermeyer M. Living with a schizophrenic patient: a comparative study of burden as it affects parents and spouses. Psychiatry. 2002;65(2):110-23.

12. Jungbauer J, Wittmund B, Dietrich S, Angermeyer MC. The disregarded caregivers: subjective burden in spouses of schizophrenia patients. Schizophr Bull. 2004;30(3):665-75.

13. Waidman MAP. 0 cuidado às famílias de portadores de transtornos mentais no paradigma da desinstitucionalização [tese]. Santa Catarina: Universidade Federal de Santa Catarina; 2004.
14. Albuquerque EPT, Bandeira M, Calzavara MGP. Sobrecarga de familiares de pacientes psiquiátricos: a influência do grau de parentesco. VI Congresso de Produção Científica da Universidade Federal de São João del-Rei; São João del-Rei. São João del- Rei, MG: Universidade Federal de São João del- Rei; 2007.

15. Martínez A, Nadal S, Beperet M, Mendióroz P. El enfermo esquizofrénico y sus familias inmersos en un cambio profundo. An Sist Sanit Navar. 2000;23(1):111-22.

16. Doornbos MM. The problems and coping methods of caregivers of young adults with mental illness. J Psychosoc Nurs Ment Health Serv. 1997;35(9):22-6.

17. Scazufca M, Menezes PR, Almeida OP. Caregiver burden in an elderly population with depression in São Paulo, Brazil. Soc Psychiatry Psychiatr Epidemiol. 2002;37(9): 416-22.

18. Garrido R, Menezes PR. Impacto em cuidadores de idosos com demência atendidos em um serviço psicogeriátrico. Rev Saude Publica. 2004;38(6):835-41.

19. Barroso SM. Sobrecarga de familiares de pacientes psiquiátricos: fatores associados [dissertação]. Belo Horizonte: Universidade Federal de Minas Gerais; 2006.

20. Selltiz C, Wrightsman LS, Cook S. Métodos de pesquisa nas relações sociais. São Paulo: EPU; 1976. Volume 1. Delineamentos de pesquisa.

21. Tessler RC, Gamache GM. The Family Burden Interview Schedule - Short Form (FBIS/SF). Armherst: Machmer Hall; 1994.

22. Bandeira M, Calzavara MGP, Varella AAB. Escala de sobrecarga dos familiares de pacientes psiquiátricos - FBIS-BR: adaptação transcultural para 0 Brasil. J Bras Psiquiatr. 2005;54(3):206-14.

23. Bandeira M, Calzavara MGP, Barroso SM, Freitas LC. Family Burden Interview Scale for Relatives of Psychiatric Patients (FBIS-BR): Reliability Study of the Brazilian Version. J Bras Psiquiatr. 2007;29(1):47-50.

24. Barroso SM, Bandeira M, Nascimento E. Fatores preditores da sobrecarga subjetiva de familiares de pacientes psiquiátricos atendidos na rede pública de Belo Horizonte, Minas Gerais, Brasil. Cad Saude Publica. 2009;25(9):1957-68.

25. Barroso SM, Bandeira M, Nascimento E. Sobrecarga de familiares de pacientes psiquiátricos atendidos na rede pública. Rev Psiq Clín. 2007;34(6):270-7.

26. St-Onge M, Lavoie F. The experience of caregiving among mothers of adults suffering from psychotic disorders: factor associated to their psychological distress. Am J Community Psychol. 1997;25(1):73-94.

27. Ruggeri M. Patients' and relatives' satisfaction with psychiatric services: the state of the art of its measurement. Soc Psychiatry Psychiatr Epidemiol. 1994;29(5):212-27.

28. Garrido R, Almeida 0. Distúrbios de comportamento em pacientes com demência: impacto sobre a vida do cuidador. Arq Neuropsiquiatr. 1999;57(2B):427-34. 